\title{
The Piltz divisor problem in number fields: An improved lower bound by Soundararajan's method
}

\author{
by \\ K. Girstmair (Innsbruck), M. Kühleitner (Wien), \\ W. Müller (Graz) and W. G. NowAK (Wien) \\ Dedicated to Professor Franz Halter-Koch on his 60th birthday
}

1. Introduction. The branch of analytic number theory which relates the size of certain arithmetic functions to the number of lattice points in certain domains has a long and very prolific history. Enlightening expositions can be found in the monographs by F. Fricker [1], E. Krätzel [11], [12], and M. N. Huxley [7]. The most classical topics of this theory are the Dirichlet divisor problem and the Gaussian circle problem: they are concerned with the error terms $\Delta(x)$ and $P(x)$ in the identities

$$
\sum_{1 \leq n \leq x} d(n)=x \log x+(2 \gamma-1) x+\Delta(x), \quad \sum_{1 \leq n \leq x} r(n)=\pi x+P(x),
$$

where $d(n)$ counts the number of divisors of $n \in \mathbb{N}$ and $r(n)$ the number of ways to write $n$ as a sum of two squares. The sharpest upper bounds to date are due to M. N. Huxley [6], [8], the present "records" reading

$$
\Delta(x) \ll x^{131 / 416}(\log x)^{26957 / 8320}, \quad P(x) \ll x^{131 / 416}(\log x)^{18637 / 8320},
$$

where $\frac{131}{416}=0.3149 \ldots$.

Concerning lower bounds, J. L. Hafner [2], [3] in 1981 improved upon G. H. Hardy's classical results [5], showing that $\left({ }^{1}\right)$

$$
\begin{aligned}
& \Delta(x)=\Omega_{+}\left(x^{1 / 4}(\log x)^{1 / 4}\left(\log _{2} x\right)^{(3+2 \log 2) / 4} \exp \left(-c_{1}\left(\log _{3} x\right)^{1 / 2}\right)\right), \\
& P(x)=\Omega_{-}\left(x^{1 / 4}(\log x)^{1 / 4}\left(\log _{2} x\right)^{(\log 2) / 4} \exp \left(-c_{2}\left(\log _{3} x\right)^{1 / 2}\right)\right),
\end{aligned}
$$

with certain $c_{1}, c_{2}>0$. Very recently, K. Soundararajan [19] developed an ingenious new method by which he sharpened these bounds (up to the am-

2000 Mathematics Subject Classification: 11N37, 11P21, 11R45.

Key words and phrases: the Piltz divisor problem, Chebotarev density theorem.

$\left({ }^{1}\right)$ For the definitions of the different $\Omega$-symbols, cf. E. Krätzel [11, p. 14]. Here and throughout, $\log _{j}$ stands for the $j$-fold iterated logarithm. 
biguity of the sign) to

$$
\begin{aligned}
& \Delta(x)=\Omega\left(x^{1 / 4}(\log x)^{1 / 4}\left(\log _{2} x\right)^{(3 / 4)\left(2^{4 / 3}-1\right)}\left(\log _{3} x\right)^{-5 / 8}\right), \\
& P(x)=\Omega\left(x^{1 / 4}(\log x)^{1 / 4}\left(\log _{2} x\right)^{(3 / 4)\left(2^{1 / 3}-1\right)}\left(\log _{3} x\right)^{-5 / 8}\right) .
\end{aligned}
$$

To visualize the refinement in the exponent of the $\log _{2} x$-factor, note that $\frac{1}{4}(3+2 \log 2)=1.0965 \ldots, \frac{1}{4} \log 2=0.1732 \ldots$, while $\frac{3}{4}\left(2^{4 / 3}-1\right)=1.1398 \ldots$, $\frac{3}{4}\left(2^{1 / 3}-1\right)=0.1949 \ldots$

The objective of the present article is to extend Soundararajan's approach to a much more general situation which includes the two classical problems as special cases. Let $K$ be an arbitrary algebraic number field of degree $k$, and denote by $\mathfrak{o}_{K}$ its ring of algebraic integers. For a positive integer $m$ we consider the arithmetic function $d_{K, m}(n)$ defined by the relation

$$
\zeta_{K}^{m}(s)=\sum_{n=1}^{\infty} d_{K, m}(n) n^{-s} \quad(\operatorname{Re}(s)>1),
$$

where $\zeta_{K}$ is the Dedekind zeta-function of $K$. In other words, $d_{K, m}(n)$ counts the number of $m$-tuples $\left(\mathfrak{n}_{1}, \ldots, \mathfrak{n}_{m}\right)$ of $\mathfrak{o}_{K}$-ideals with $N_{K}\left(\mathfrak{n}_{1} \cdots \mathfrak{n}_{m}\right)=n$. Here $N_{K}(\mathfrak{n})$ denotes the absolute norm of $\mathfrak{n}$. In analogy to (1), we are interested in the behavior of the error term in the identity

$$
\sum_{1 \leq n \leq x} d_{K, m}(n)=\underset{s=1}{\operatorname{res}}\left(\zeta_{K}^{m}(s) \frac{x^{s}}{s}\right)+\Delta_{K, m}(x)
$$

as $x \rightarrow \infty$. The investigation of $\Delta_{\mathbb{Q}, m}$ is known as the Piltz divisor problem. Concerning upper bounds for $\Delta_{K, m}(x)$, see A. Ivić [9] (where the rational case is discussed in detail), W. G. Nowak [16], and also the references in W. Narkiewicz [15, Ch. 7].

In this article we apply Soundararajan's method to establish a sharp lower estimate for $\Delta_{K, m}(x)$. To describe the result we have to introduce some notations. For $0 \leq \nu \leq k$ let $P_{\nu}$ denote the set of all rational primes which are unramified in $K$, and which are divisible by exactly $\nu \mathfrak{o}_{K}$-prime ideals of degree 1 . The $P_{\nu}$ are disjoint and together with the finitely many ramified primes exhaust the rational primes. Our result depends on the Dirichlet densities $\delta_{\nu}$ of the sets $P_{\nu}$. These densities can be calculated as follows. Let $L$ be the minimal normal extension of $K, G=\operatorname{Gal}(L / \mathbb{Q})$ its Galois group, and $H=\operatorname{Gal}(L / K)$ the subgroup of $G$ corresponding to $K$ via Galois theory. Then

$$
\delta_{\nu}=|G|^{-1}\left|\left\{\tau \in G:\left|\left\{\sigma \in G: \tau \in \sigma H \sigma^{-1}\right\}\right|=\nu|H|\right\}\right| .
$$

The constants $\delta_{\nu}$ satisfy $\sum_{\nu=1}^{k} \nu \delta_{\nu}=1$. If $K$ is normal, then $P_{\nu}$ is empty for $1 \leq \nu<k$. Hence $\delta_{\nu}=0$ for $1 \leq \nu<k$ and $\delta_{k}=1 / k$ in this case. Additionally, denote by $R$ the number of $1 \leq \nu \leq k$ with $\delta_{\nu}>0$. 
TheOREM 1. Let $k m>1$. Then for $x \rightarrow \infty$ the error term $\Delta_{K, m}$ in $(3)$ satisfies

$$
\Delta_{K, m}(x)=\Omega\left((x \log x)^{\frac{k m-1}{2 k m}}\left(\log _{2} x\right)^{\kappa}\left(\log _{3} x\right)^{-\lambda}\right)
$$

where

$$
\kappa=\frac{k m+1}{2 k m}\left(\sum_{\nu=1}^{k} \delta_{\nu}(\nu m)^{\frac{2 k m}{k m+1}}-1\right), \quad \lambda=\frac{k m+1}{4 k m} R+\frac{k m-1}{2 k m} .
$$

Furthermore, let $r_{1}$ denote the number of real conjugates of $K$. If $m r_{1} \equiv 3$ (mod 8$)$ then $(5)$ remains true if $\Omega$ is replaced by $\Omega_{+}$. If $m r_{1} \equiv 7(\bmod 8)$ then (5) remains true if $\Omega$ is replaced by $\Omega_{-}$.

This theorem contains (2) as special cases. The Dirichlet divisor problem corresponds to $K=\mathbb{Q}$ and $m=2$, and the circle problem corresponds to $K=\mathbb{Q}(i)$ and $m=1$. In Section 4 we discuss further special examples. Theorem 1 should be compared with the classical estimate

$$
\Delta_{K, m}(x)=\Omega_{*}\left((x \log x)^{\frac{k m-1}{2 k m}}\left(\log _{2} x\right)^{m-1}\right)
$$

due to P. Szegő and A. Walfisz [20], [21], and with Hafner's [4] refinement

$$
\Delta_{K, m}(x)=\Omega_{*}\left((x \log x)^{\frac{k m-1}{2 k m}}\left(\log _{2} x\right)^{\kappa^{\prime}} \exp \left(-c_{3}\left(\log _{3} x\right)^{1 / 2}\right)\right) .
$$

Here $c_{3}>0$ is a constant (depending on $K$ and $m$ ) and

$$
\kappa^{\prime}=\frac{k m-1}{2 k m}\left(m \log m+\left(\sum_{\nu=1}^{k} \delta_{\nu} \nu \log \nu\right) m-m+1\right)+m-1 .
$$

In both of these last $\Omega$-statements,

$$
\Omega_{*}= \begin{cases}\Omega_{ \pm} & \text {if } k m \geq 4 \text { or } K \text { is cubic and not totally real, } \\ \Omega_{-} & \text {if } m=1 \text { and } K \text { is quadratic imaginary, } \\ \Omega_{+} & \text {if } m=2,3 \text { and } K=\mathbb{Q} \\ & \text { or } m=1, k=2,3 \text { and } K \text { is totally real. }\end{cases}
$$

Soundararajan's method in most cases fails to control the sign of the large values exhibited. But note that always $\kappa>\kappa^{\prime}$. For $k$ fixed and $m \rightarrow \infty$, we see that $\kappa$ roughly grows like $c m^{2}$ with $c=\frac{1}{2} \sum_{\nu=1}^{k} \nu^{2} \delta_{\nu}$, while Hafner's $\kappa^{\prime}$ only behaves like $\frac{1}{2} m \log m$.

Soundararajan gives a heuristic reason why the exponent of $\log _{2} x$ in (2) may be best possible. His argument carries over to our more general situation. Arrange the sequence $d_{K, m}(n) n^{-\frac{k m-1}{2 k m}}$ in descending order, and let $S(M)$ denote the sum of the first $M$ largest values. Then an optimal Omega result is expected to be of the form $\Delta_{K, m}(n)=\Omega\left(x^{\frac{k m-1}{2 k m}} S(\log x)\right)$. It can be proved that $S(M)=M^{\frac{k m-1}{2 k m}}(\log M)^{\kappa+o(1)}$; thus Theorem 1 yields the expected maximal order of $\Delta_{K, m}(x)$ up to a factor $\left(\log _{2} x\right)^{o(1)}$. 
The proof of Theorem 1 runs along the same lines as in Soundararajan's paper [19]. We start with the known asymptotic expansion of a Borel mean value of $\Delta_{K, m}$. To this we apply Soundararajan's key lemma (Lemma 1 below) to deduce the Omega result. In order to do this we have to count the natural numbers $n \leq x$ which have exactly $r_{\nu}$ distinct prime divisors in the set $P_{\nu}$ for $1 \leq \nu \leq k$ and no prime divisors which ramify in $K$. Moreover, we need a result which is uniform in $r=r_{1}+\cdots+r_{k} \leq B \log _{2} x$. Here $B \geq 1$ is a given constant. What we need is a special case of the following theorem (it may be viewed as a generalization of the Chebotarev density theorem). Note that for a prime $p$, which is unramified in $K$, the type of the prime ideal decomposition of $p \mathfrak{o}_{K}$ in $K$ is determined by $H=\operatorname{Gal}(L / K)$ and the Frobenius conjugacy class of $p$ in $L / \mathbb{Q}$ (see Section 2).

Let $Q$ be an algebraic number field, $K$ an algebraic extension of $Q$, and $L$ an algebraic extension of $K$ which is normal over $Q$. Set $G=\operatorname{Gal}(L / Q)$ and $H=\operatorname{Gal}(L / K)$. For every prime ideal $\mathfrak{p}$ in $\mathfrak{o}_{Q}$ denote by $(\mathfrak{p}, L / Q)$ the Frobenius conjugacy class of $\mathfrak{p}$ in $L / Q$ (see Section 3 ).

TheOREM 2. Let $G=\bigcup_{\nu=1}^{d} A_{\nu}$ be a partition of $G$ into $d$ sets $A_{\nu} \neq \emptyset$ which are unions of conjugacy classes of $G$. For $r_{\nu} \geq 0,1 \leq \nu \leq d$, denote by $\pi_{K / Q}\left(x, r_{1}, \ldots, r_{d}\right)$ the number of $\mathfrak{o}_{Q}$-ideals $\mathfrak{n}$ with $N_{Q}(\mathfrak{n}) \leq x$, such that $\mathfrak{n}$ has no prime divisor which is ramified in $L / Q$, and $r_{\nu}$ distinct prime divisors $\mathfrak{p}$ with $(\mathfrak{p}, L / Q) \subseteq A_{\nu}$ for $1 \leq \nu \leq d$. For given $B \geq 1$ and $r=\sum_{\nu=1}^{d} r_{\nu}$, we have, uniformly in $1 \leq r \leq B \log _{2} x$ and $x \geq 3$,

$$
\begin{aligned}
& \pi_{K / Q}\left(x, r_{1}, \ldots, r_{d}\right) \\
& \quad=\left(\prod_{\nu=1}^{d} \frac{\delta_{\nu}^{r_{\nu}}}{r_{\nu} !}\right) \frac{r x}{\log x}\left(\log _{2} x\right)^{r-1}\left(\mu\left(x, r_{1}, \ldots, r_{d}\right)+O\left(\frac{r}{\left(\log _{2} x\right)^{2}}\right)\right) .
\end{aligned}
$$

Here $\delta_{\nu}=\left|A_{\nu}\right| /|G|$ denotes the density of $A_{\nu}$, and

$$
\mu\left(x, r_{1}, \ldots, r_{d}\right)=H\left(\frac{(r-1) r_{1}}{r \delta_{1} \log _{2} x}, \ldots, \frac{(r-1) r_{d}}{r \delta_{d} \log _{2} x}\right),
$$

where $H$ is the function defined in (24). Furthermore, uniformly for $1 \leq r \leq$ $B \log _{2} x$ and $x \geq 3$,

$$
\mu\left(x, r_{1}, \ldots, r_{d}\right)=1+O\left(\frac{r}{\log _{2} x}\right), \quad \mu\left(x, r_{1}, \ldots, r_{d}\right) \asymp 1 .
$$

COROllary. Let $q \geq 1$, and let $a_{1}, \ldots, a_{d}$ be d integers which are incongruent modulo $q$ and relatively prime to $q$. Denote by $\pi\left(x, r_{1}, \ldots, r_{d}\right)$ the number of integers $n \leq x$ which have $r_{\nu}$ distinct prime divisors $p \equiv a_{\nu}$ $(\bmod q)$ for $1 \leq \nu \leq d$, and no other ones. For given $B \geq 1$, and $r=$ $\sum_{\nu=1}^{d} r_{\nu}$, we have, uniformly in $1 \leq r \leq B \log _{2} x$ and $x \geq 3$, 


$$
\begin{aligned}
\pi\left(x, r_{1}, \ldots, r_{d}\right) & =\frac{r}{r_{1} ! \ldots r_{d} ! \varphi(q)^{r}} \frac{x}{\log x}\left(\log _{2} x\right)^{r-1}\left(1+O\left(\frac{r}{\log _{2} x}\right)\right), \\
\pi\left(x, r_{1}, \ldots, r_{d}\right) & \asymp \frac{r}{r_{1} ! \ldots r_{d} ! \varphi(q)^{r}} \frac{x}{\log x}\left(\log _{2} x\right)^{r-1} .
\end{aligned}
$$

Proof. The Corollary is a special case of Theorem 2. Set $Q=\mathbb{Q}$ and let $K$ be the cyclotomic field $\mathbb{Q}\left(\zeta_{q}\right)$. Here $\zeta_{q}$ denotes a primitive root of unity of order $q . K / \mathbb{Q}$ is normal and $|\operatorname{Gal}(K / \mathbb{Q})|=\varphi(q)$. A prime $p$ is ramified in $K / \mathbb{Q}$ if and only if $p \mid q$. For an integer $a$ with $(a, q)=1$, let $\sigma_{a} \in \operatorname{Gal}(K / \mathbb{Q})$ be uniquely defined by $\sigma_{a}\left(\zeta_{q}\right)=\zeta_{q}^{a}$. For $p \nmid q$ it is well known that $(p, K / \mathbb{Q})=\left\{\sigma_{a}\right\}$ if and only if $p \equiv a(\bmod q)$ (cf. G. J. Janusz $[10$, p. 104]). This proves the Corollary.

2. Proof of Theorem 1. We start with the asymptotic expansion for the Borel mean value of the error term $\Delta_{K, m}(t)$. For real parameters $T \geq 40$ and $t \in\left[T / 2, T^{2}\right]$, and arbitrary $\varepsilon>0$,

$$
\begin{aligned}
B(t) & :=\frac{1}{\Gamma(h+1)} \int_{0}^{\infty} e^{-u} u^{h} \Delta_{K, m}\left(X u^{k m / 2}\right) d u \\
& =c_{0} t^{(k m-1) / 2} S(t)+O\left(t^{k m / 2-3 / 4+\varepsilon}\right)
\end{aligned}
$$

where

$$
S(t)=\sum_{n \leq T^{\varepsilon_{0}}} d_{K, m}(n) n^{\theta-1} \exp \left(-c_{2}(n X)^{2 /(k m)}\right) \cos \left(2 \pi c_{1} n^{1 /(k m)} t+\beta_{0}\right)
$$

with

$$
\begin{aligned}
X:=(\log T)^{-2}, & h=h(t):=\left(t X^{-1 /(k m)}\right)^{2}, \\
\theta & =\frac{k m-1}{2 k m}, \quad \beta_{0}=-\frac{3 \pi}{4}+\frac{\pi}{4} m r_{1} .
\end{aligned}
$$

Here $\varepsilon_{0}>0$ is a sufficiently small constant, $c_{j}$ are positive constants which depend only on the field $K$ and on $m$. This asymptotic expansion goes back to G. Szegö and A. Walfisz [20], [21]. For a more recent treatment in the context of asymmetric divisor problems, see W. G. Nowak [17, p. 269].

The following lemma is due to K. Soundararajan [19]. It gives a general lower bound for trigonometric series. Let $(f(n))_{n \geq 1}$ be a sequence of non-negative real numbers and $\left(\lambda_{n}\right)_{n \geq 1}$ be a non-decreasing sequence of non-negative real numbers. Suppose that $\sum_{n \geq 1} f(n)<\infty$ and consider the trigonometric series

$$
F(t):=\sum_{n \geq 1} f(n) \cos \left(2 \pi \lambda_{n} t+\beta\right)
$$

where $\beta \in \mathbb{R}$. 
Lemma 1. Let $L \geq 2$ and $N \geq 1$ be integers. Let $\mathcal{M}$ be a set of integers such that $\lambda_{m} \in\left[\lambda_{N} / 2,3 \lambda_{N} / 2\right]$ for each $m \in \mathcal{M}$. For any $T \geq 2$ there exists a point $t \in\left[T / 2,(6 L)^{|\mathcal{M}|+1} T\right]$ such that

$$
|F(t)| \geq \frac{1}{8} \sum_{m \in \mathcal{M}} f(m)-\frac{1}{L-1} \sum_{\substack{n \\ \lambda_{n} \leq 2 \lambda_{N}}} f(n)-\frac{4}{\pi^{2} X \lambda_{N}} \sum_{n \geq 1} f(n) .
$$

If $\beta \equiv 0(\bmod 2 \pi)$ then the conclusion $(7)$ holds with $F(t)$ in place of $|F(t)|$. If $\beta \equiv \pi(\bmod 2 \pi)$ then the conclusion $(7)$ holds with $-F(t)$ in place of $|F(t)|$.

To prove Theorem 1 we apply Lemma 1 with

$$
f(n)=d_{K, m}(n) n^{\theta-1} \exp \left(-c_{2}(n X)^{2 /(k m)}\right)
$$

if $n \leq T^{\varepsilon_{0}}$ and $f(n)=0$ else, $\lambda_{n}=c_{1} n^{1 /(k m)}$ and $\beta=\beta_{0}$. For $0 \leq \nu \leq k$ let $P_{\nu}$ be the set of all rational primes which are unramified in $K$ and which are divisible by exactly $\nu \mathfrak{o}_{K}$-prime ideals of degree 1 . Let $I=\{1 \leq \nu \leq k$ : $\left.\delta_{\nu}>0\right\}$, where $\delta_{\nu}$ denotes the Dirichlet density of $P_{\nu}$ as in the introduction. Then $R=|I|$.

We choose $\mathcal{M}$ to be the set of all $n \in\left[2^{-k m} N,(3 / 2)^{k m} N\right]$ such that $n$ has $r_{\nu}$ distinct prime factors from $P_{\nu}$ for all $\nu \in I$ and no others. Here $r_{\nu}=\left[\lambda_{\nu} \log _{2} N\right]$ with some parameters $\lambda_{\nu}>0$ (the optimal choice turns out to be $\left.\lambda_{\nu}=\delta_{\nu}(\nu m)^{2 k m /(k m+1)}\right)$.

For $n \in \mathcal{M}$ the value of $d_{K, m}(n)$ is large. Indeed, if $p \in P_{\nu}$ is prime then the factorization of $p \mathfrak{o}_{K}$ contains exactly $\nu$ prime ideals $\mathfrak{p}$ with $N_{K}(\mathfrak{p})=p$. Hence $d_{K, 1}(p)=\nu$. Since for every prime ideal $\mathfrak{p}$ there are $m$ tuples $\left(\mathfrak{n}_{1}, \ldots, \mathfrak{n}_{m}\right)$ with $N_{K}\left(\mathfrak{n}_{1} \cdots \mathfrak{n}_{m}\right)=p$ (take one of the $\mathfrak{n}_{i}$ equal to $\mathfrak{p}$, all others equal to $\left.\mathfrak{o}_{K}\right)$ we find $d_{K, m}(p)=\nu m$. The same argument proves $d_{K, m}\left(p^{e}\right) \geq \nu m$ for $e \geq 1$ and $p \in P_{\nu}$. Hence

$$
d_{K, m}(n) \geq \prod_{\nu \in I}(\nu m)^{r_{\nu}} \quad(n \in \mathcal{M}) .
$$

Next we use Theorem 2 to estimate the cardinality of $\mathcal{M}$. Let $L$ denote the minimal normal extension of $K$. A rational prime $p$ is ramified in $K$ if and only if $p$ is ramified in $L$ (cf. W. Narkiewicz [15, Cor. 2 to Prop. 4.12]). For an unramified $p$ let $(p, L / \mathbb{Q})$ be the Frobenius symbol of $p$ in $L / \mathbb{Q}$. This is a class of conjugate elements of $G=\operatorname{Gal}(L / \mathbb{Q})$. Together with $H=\operatorname{Gal}(L / K)$ it determines the type of factorization of $p$ (unramified) in the following way (cf. G. J. Janusz [10, Prop. 2.8]). Take any $\varphi \in(p, L / \mathbb{Q})$. If the set of right cosets of $H$ in $G$ is partitioned into sets

$$
\left\{H \sigma_{1}, H \sigma_{1} \varphi, \ldots, H \sigma_{1} \varphi^{f_{1}-1}\right\}, \ldots,\left\{H \sigma_{j}, H \sigma_{j} \varphi, \ldots, H \sigma_{j} \varphi^{f_{j}-1}\right\}
$$

with $\sigma_{i} \in G$ and $f_{i} \geq 1$, then $p \mathfrak{o}_{K}=\mathfrak{p}_{1} \cdots \mathfrak{p}_{j}$. Here $\mathfrak{p}_{i}$ are prime ideals in $\mathfrak{o}_{K}$ with initial degree $f\left(\mathfrak{p}_{i} \mid \mathbb{Q}\right)=f_{i}$, thus $N_{K}\left(\mathfrak{p}_{i}\right)=p^{f_{i}}$. Hence $p \in P_{\nu}$ if and only if $p$ is unramified in $K$, and there are exactly $\nu$ right cosets $H \sigma$ with 
$H \sigma \varphi=H \sigma$. In other words

$$
P_{\nu}=\left\{p \text { unramified in } K:\left|\left\{\sigma \in G: \varphi \in \sigma^{-1} H \sigma\right\}\right|=\nu|H|\right\} .
$$

The set on the right hand side is independent of the choice of $\varphi \in(p, L / \mathbb{Q})$. Let

$$
A_{\nu}=\left\{\tau \in G:\left|\left\{\sigma \in G: \tau \in \sigma^{-1} H \sigma\right\}\right|=\nu|H|\right\} .
$$

Then $p \in P_{\nu}$ if and only if $(p, L / \mathbb{Q}) \subseteq A_{\nu}$. The density of $A_{\nu}$ is $\delta_{\nu}=\left|A_{\nu}\right| /|G|$. This explains (4). If we write $c_{1}=(1 / 2)^{k m}, c_{2}=(3 / 2)^{k m}$ for the moment, Theorem 2 implies (with the choice of the numbers $r_{\nu}$ made earlier)

$$
\begin{aligned}
|\mathcal{M}| \asymp & r\left(\prod_{\nu \in I} \frac{\delta_{\nu}^{r_{\nu}}}{r_{\nu} !}\right)\left(H\left(\frac{(r-1) r_{1}}{r \delta_{1} \log _{2}\left(c_{2} N\right)}, \ldots, \frac{(r-1) r_{d}}{r \delta_{d} \log _{2}\left(c_{2} N\right)}\right)\right. \\
& \times \frac{c_{2} N}{\log \left(c_{2} N\right)}\left(\log _{2}\left(c_{2} N\right)\right)^{r-1} \\
& \left.-H\left(\frac{(r-1) r_{1}}{r \delta_{1} \log _{2}\left(c_{1} N\right)}, \ldots, \frac{(r-1) r_{d}}{r \delta_{d} \log _{2}\left(c_{1} N\right)}\right) \frac{c_{1} N}{\log \left(c_{1} N\right)}\left(\log _{2}\left(c_{1} N\right)\right)^{r-1}\right) .
\end{aligned}
$$

For $N$ large, the arguments of $H$ here differ only by $o(1)$ in each component, hence the same is true for the two values of $H$ involved. Observe that they are also $\asymp 1$. However,

$$
\frac{c_{2} N}{\log \left(c_{2} N\right)}\left(\log _{2}\left(c_{2} N\right)\right)^{r-1}-\frac{c_{1} N}{\log \left(c_{1} N\right)}\left(\log _{2}\left(c_{1} N\right)\right)^{r-1} \asymp \frac{N}{\log N}\left(\log _{2} N\right)^{r-1} .
$$

Therefore,

$$
|\mathcal{M}| \asymp r\left(\prod_{\nu \in I} \frac{\delta_{\nu}^{r_{\nu}}}{r_{\nu} !}\right) \frac{N}{\log N}\left(\log _{2} N\right)^{r-1} \asymp \frac{N}{\log N} \prod_{\nu \in I} \frac{\left(\delta_{\nu} \log _{2} N\right)^{r_{\nu}}}{r_{\nu} !} .
$$

Using $n ! \sim \sqrt{2 \pi} n^{n+1 / 2} e^{-n}$ we find

$$
|\mathcal{M}| \asymp N(\log N)^{\alpha}\left(\log _{2} N\right)^{\beta},
$$

where $\alpha=-1+\sum_{\nu \in I} \lambda_{\nu}\left(1+\log \delta_{\nu}-\log \lambda_{\nu}\right)$ and $\beta=-R / 2$.

To complete the proof of Theorem 1 , let $T \geq 40$ be a real parameter, and choose $L=\left\lceil\left(\log _{2} T\right)^{m}\right\rceil$ and

$$
N=\left\lceil c \log T\left(\log _{2} T\right)^{-\alpha}\left(\log _{3} T\right)^{-1-\beta}\right\rceil .
$$

Here $c$ denotes a positive constant, which we choose sufficiently small to ensure

$$
(6 L)^{|\mathcal{M}|+1} \leq T .
$$

Moreover, this choice implies $n X \ll 1$ for $n \ll N$ and $t \in\left[\frac{1}{2} T, T^{2}\right]$. By (8)-(10) we find 


$$
\begin{aligned}
\sum_{n \in \mathcal{M}} f(n) & \gg N^{\theta-1}|\mathcal{M}| \prod_{\nu \in I}(\nu m)^{r_{\nu}} \gg N^{\theta}(\log N)^{\alpha+\sum_{\nu \in I} \lambda_{\nu} \log (\nu m)}\left(\log _{2} N\right)^{\beta} \\
& \gg(\log T)^{\theta}\left(\log _{2} T\right)^{(1-\theta) \alpha+\sum_{\nu \in I} \lambda_{\nu} \log (\nu m)}\left(\log _{3} T\right)^{(1-\theta) \beta-\theta} .
\end{aligned}
$$

The choice $\lambda_{\nu}=\delta_{\nu}(\nu m)^{2 k m /(k m+1)}, \nu \in I$, maximizes the exponent of $\log _{2} T$. This yields

$$
\sum_{m \in \mathcal{M}} f(n) \gg(\log T)^{\theta}\left(\log _{2} T\right)^{\kappa}\left(\log _{3} T\right)^{-\lambda}
$$

with $\kappa$ and $\lambda$ as in Theorem 1 .

It remains to show that the other two terms on the right hand side of $(7)$ are small. The bound $\sum_{n \leq x} d_{K, m}(n) \ll x(\log x)^{m-1}$ together with partial summation yields $\sum_{\lambda_{n} \leq \lambda_{N}} f(n) \ll N^{\theta}(\log N)^{m-1}$. After division by $L-1$ this is small compared to the right hand side of (12). Similarly, for $t \in\left[\frac{1}{2} T, T^{2}\right]$,

$$
\frac{4}{\pi^{2} T \lambda_{N}} \sum_{n=1}^{\infty} f(n) \ll \frac{1}{T N} \sum_{1 \leq n \leq T^{\varepsilon_{0}}} d_{K, m}(n) n^{\theta-1} \ll T^{3 \varepsilon_{0}-1} .
$$

This is again small compared with (12). Using (7) and (11) we conclude that for arbitrary $T \geq 40$, there exists a value $t \in\left[\frac{1}{2} T, T^{2}\right]$ with

$$
|B(t)| \gg t^{(k m-1) / 2}(\log t)^{\theta}\left(\log _{2} t\right)^{\kappa}\left(\log _{3} t\right)^{-\lambda} .
$$

Now let us assume that (5) is false. Then for every $\varepsilon_{1}>0$ there is a constant $c$ such that, for all $u>0$,

$$
\left|\Delta_{K, m}(u)\right| \leq c+\varepsilon_{1} u^{\theta} \mathcal{L}(u),
$$

where

$$
\mathcal{L}(u):=(\log u)^{\theta}\left(\log _{2} u\right)^{\kappa}\left(\log _{3} u\right)^{-\lambda}
$$

for $u \geq 20$, and $\mathcal{L}(u)=\mathcal{L}(20)$ else. By the definition (6) of $B(t)$, this implies that

$$
|B(t)| \leq c+\frac{\varepsilon_{1}}{\Gamma(h+1)} \int_{0}^{\infty} e^{-u} u^{h}\left(X u^{k m / 2}\right)^{\theta} \mathcal{L}\left(X u^{k m / 2}\right) d u
$$

for all $t>0$. Estimating this integral by Hafner's Lemma 2.3.6 in [3], we obtain

$$
|B(t)| \leq c+c^{*} \varepsilon_{1}\left(X h^{k m / 2}\right)^{\theta} \mathcal{L}\left(X h^{k m / 2}\right)=c+c^{*} \varepsilon_{1} t^{(k m-1) / 2} \mathcal{L}\left(t^{k m / 2}\right) .
$$

Together with (13), this yields a positive lower bound for $\varepsilon_{1}$. This proves (5). If $\beta_{0}$ is an integer multiple of $\pi$, Lemma 1 yields (13) with $|B(t)|$ replaced by $B(t)$ or $-B(t)$. Completing the argument as before, we obtain, for this case, the more precise information stated in Theorem 1. 
3. Proof of Theorem 2. We use a variant of the method of SelbergDelange to prove Theorem 2. For a description of this method see G. Tenenbaum [22]. Let $\mathfrak{d}$ be the relative discriminant of $L / Q$. An $\mathfrak{o}_{Q}$-prime ideal $\mathfrak{p}$ is ramified in $L / Q$ if and only if $\mathfrak{p} \mid \mathfrak{d}$. For an $\mathfrak{o}_{Q}$-ideal $\mathfrak{n}$ denote by $\omega_{\nu}(\mathfrak{n})$ the number of distinct $\mathfrak{o}_{Q}$-prime ideal divisors $\mathfrak{p}$ of $\mathfrak{n}$ which are unramified in $L / Q$ and which satisfy $(\mathfrak{p}, L / Q) \subseteq A_{\nu}$. For $s \in \mathbb{C}$ and $z=\left(z_{1}, \ldots, z_{d}\right) \in \mathbb{C}^{d}$ consider the Dirichlet series

$$
F(s, z)=\sum_{(\mathfrak{n}, \mathfrak{d})=1}\left(\prod_{\nu=1}^{d} z_{\nu}^{\omega_{\nu}(\mathfrak{n})}\right) N_{Q}(\mathfrak{n})^{-s} \quad(\operatorname{Re}(s)>1) .
$$

The sum runs over all $\mathfrak{o}_{Q}$-ideals $\mathfrak{n}$ relatively prime to $\mathfrak{d}$. It is absolutely convergent in the half-plane $\operatorname{Re}(s)>1$. Since $z_{\nu}^{\omega_{\nu}(\mathfrak{n})}$ is multiplicative in $\mathfrak{n}$ we find

$$
F(s, z)=\prod_{\nu=1}^{d} \prod_{\substack{\mathfrak{p} \nmid \mathfrak{d} \\(\mathfrak{p}, L / Q) \subseteq A_{\nu}}}\left(1+\frac{z_{\nu}}{N_{Q}(\mathfrak{p})^{s}-1}\right) .
$$

The plan of the proof is as follows. After the analytic continuation of $F$, standard methods give the asymptotic behavior of

$$
S(x, z)=\sum_{\substack{N_{Q}(\mathfrak{n}) \leq x \\(\mathfrak{n}, \mathfrak{d})=1}} \prod_{\nu=1}^{d} z_{\nu}^{\omega_{\nu}(\mathfrak{n})} .
$$

Then Theorem 2 follows by a $d$-fold application of Cauchy's theorem.

To study the analytic properties of $F$ we use Artin L-series (see E. de Shalit [18] for a recent survey of Artin L-series). They are defined as follows. Let $L / Q$ be a finite normal extension of $Q$ with Galois group $G$. For a prime ideal $\mathfrak{P}$ of $\mathfrak{o}_{L}$ lying over the prime ideal $\mathfrak{p}$ of $\mathfrak{o}_{Q}$, one defines the decomposition group of $\mathfrak{P}$ by

$$
D_{\mathfrak{P}}=\{\tau \in G: \tau(\mathfrak{P})=\mathfrak{P}\}
$$

and the inertia subgroup by

$$
I_{\mathfrak{P}}=\left\{\tau \in G: \tau(x) \equiv x(\bmod \mathfrak{P}) \text { for all } x \in \mathfrak{o}_{L}\right\} .
$$

The map $D_{\mathfrak{P}} \rightarrow \operatorname{Gal}\left(L_{\mathfrak{P}} / Q_{\mathfrak{p}}\right)$, induced by the restriction of $\tau \in D_{\mathfrak{P}}$ to $\mathfrak{o}_{L}$, is surjective with kernel $I_{\mathfrak{P}}$. Here $L_{\mathfrak{P}}$ and $Q_{\mathfrak{p}}$ denote the residue class fields. The Galois group $\operatorname{Gal}\left(L_{\mathfrak{P}} / Q_{\mathfrak{p}}\right)$ is cyclic with generator $x \mapsto x^{N_{Q}(\mathfrak{p})}$. Every element $\sigma_{\mathfrak{P}} \in D_{\mathfrak{P}}$ which maps to this generator is called a Frobenius element of $\mathfrak{P}$ in $L / Q$. It is only unique modulo $I_{\mathfrak{P}}$. The ideal $\mathfrak{p}$ is unramified if and only if $I_{\mathfrak{P}}=1$. In this case the Frobenius element is unique. For unramified $\mathfrak{p}$ the Frobenius elements $\sigma_{\mathfrak{P}}$ of all $\mathfrak{P} \mid \mathfrak{p}$ are conjugate. This conjugacy class is called the Frobenius conjugacy class of $\mathfrak{p}$ in $L / Q$ and denoted by $(\mathfrak{p}, L / Q)$. 
Let $V$ be a finite-dimensional $\mathbb{C}$-vector space and $\varrho: G \rightarrow \operatorname{GL}(V)$ a representation of $G$ with character $\chi$. Furthermore, denote by $V_{\mathfrak{P}}=$ $\left\{x \in V: \varrho(\sigma)(x)=x\right.$ for all $\left.\sigma \in I_{\mathfrak{P}}\right\}$ the vector space of all $I_{\mathfrak{P}}$-fixed points. Note that $V_{\mathfrak{P}}=V$ if $\mathfrak{p}$ is unramified in $L / Q$. The Artin L-series is defined as

$$
L(s, \chi)=\prod_{\mathfrak{p}} \operatorname{det}\left(\left.\left(\operatorname{Id}_{V}-\varrho\left(\sigma_{\mathfrak{P}}\right) N_{Q}(\mathfrak{p})^{-s}\right)\right|_{V_{\mathfrak{P}}}\right)^{-1} \quad(\operatorname{Re}(s)>1) .
$$

The product runs over all prime ideals $\mathfrak{p}$ in $\mathfrak{o}_{Q}$. It is absolutely and uniformly convergent in every compact subset of the half-plane $\operatorname{Re}(s)>1$. Hence $L(s, \chi)$ is holomorphic in this domain. Moreover,

$$
\log L(s, \chi)=\sum_{\mathfrak{p}} \sum_{k \geq 1} \frac{\tilde{\chi}\left(\sigma_{\mathfrak{p}}^{k}\right)}{k N_{Q}(\mathfrak{p})^{s}} \quad(\operatorname{Re}(s)>1),
$$

where

$$
\widetilde{\chi}\left(\sigma_{\mathfrak{P}}^{k}\right):=\frac{1}{\left|I_{\mathfrak{P}}\right|} \sum_{\alpha \in I_{\mathfrak{P}}} \chi\left(\sigma_{\mathfrak{P}}^{k} \alpha\right)
$$

Here and in the following, log always denotes that branch of the logarithm which is real on the positive real axis. If $\mathfrak{p}$ is unramified, e.g. $\mathfrak{p} \nmid \mathfrak{d}$, then $\tilde{\chi}\left(\sigma_{\mathfrak{P}}^{k}\right)=\chi\left(\sigma_{\mathfrak{P}}^{k}\right)$. The Artin L-series can be continued meromorphically to the entire complex plane. There is a real constant $c=c(L)>0$ such that, for all irreducible characters $\chi$,

$$
L(s, \chi) \neq 0 \quad \text { for } s \in \mathcal{D}:=\{\sigma+i t: \sigma \geq \psi(t)\},
$$

where $\psi(t):=1-c / \log (|t|+3)$. Moreover, $L(s, \chi)$ is holomorphic in $\mathcal{D}$ (up to a simple pole at $s=1$ if $\chi$ is the trivial character) and, for $s \in \mathcal{D}$ with $|t| \geq \delta>0$

$$
\log L(s, \chi) \ll \log _{2}(|t|+3) .
$$

This is well known for Hecke L-series: For a proof of (17) see J. C. Lagarias and A. M. Odlyzko [13, Chapter 8]. Using (5.9) of [13], the bound (18) is readily verified on classical lines (cf. G. Tenenbaum [22, II, Theorem 16]). If one knows (17) and (18) for Hecke L-series, both assertions follow for general Artin L-series, since every Artin L-series can be expressed as a quotient of products of Hecke L-series (this is a consequence of the Brauer induction theorem). Note that we do not try to give results which are uniform in the field $L$.

For $\operatorname{Re}(s)>1$, define

$$
Z_{A}(s)=\prod_{\substack{\mathfrak{p} \nmid \mathfrak{d} \\(\mathfrak{p}, L / Q) \subseteq A}}\left(1-N_{Q}(\mathfrak{p})^{-s}\right)^{-1} .
$$


Here $A=\bigcup_{j \in J} C_{j}$ is a non-empty disjoint union of conjugacy classes $C_{j}$ of $G$. From the representation theory of finite groups we use the following facts. If $G$ splits into $h$ conjugacy classes $C_{1}, \ldots, C_{h}$, then there are exactly $h$ irreducible representations, whose characters we denote by $\chi_{1}, \ldots, \chi_{h}$. The $\chi_{i}$ are constant on every conjugacy class. They satisfy the orthogonality relations

$$
|G|^{-1} \sum_{s \in G} \chi_{i}(s) \chi_{j}\left(s^{-1}\right)= \begin{cases}1 & \text { if } i=j, \\ 0 & \text { else. }\end{cases}
$$

This implies that the indicator function $I_{A}$ of $A$ can be written as $I_{A}=$ $\sum_{i=1}^{h} \alpha_{i}(A) \chi_{i}$, where $\alpha_{i}(A)=|G|^{-1} \sum_{j \in J} \chi_{i}\left(C_{j}^{-1}\right)\left|C_{j}\right|$. Together with (16) this gives

$$
\begin{aligned}
& \sum_{i=1}^{h} \alpha_{i}(A) \log L\left(s, \chi_{i}\right) \\
& \quad=\sum_{\substack{\mathfrak{p} \nmid \mathfrak{d} \\
(\mathfrak{p}, L / Q) \subseteq A}} N_{Q}(\mathfrak{p})^{-s}+\sum_{i=1}^{h} \alpha_{i}(A)\left\{\sum_{\mathfrak{p} \mid \mathfrak{d}} \frac{\widetilde{\chi}_{i}\left(\sigma_{\mathfrak{P}}\right)}{N_{Q}(\mathfrak{p})^{s}}+\sum_{\mathfrak{p}} \sum_{k \geq 2} \frac{\widetilde{\chi}_{i}\left(\sigma_{\mathfrak{P}}^{k}\right)}{k N_{Q}(\mathfrak{p})^{k s}}\right\} .
\end{aligned}
$$

On the other hand, for $\operatorname{Re}(s)>1$,

$$
\log Z_{A}(s)=\sum_{\substack{\mathfrak{p} \nmid \mathfrak{d} \\(\mathfrak{p}, L / Q) \subseteq A}} N_{Q}(\mathfrak{p})^{-s}+\sum_{\substack{\mathfrak{p} \nmid \mathfrak{d} \\(\mathfrak{p}, L / Q) \subseteq A}} \sum_{k \geq 2} \frac{1}{k N_{Q}(\mathfrak{p})^{k s}} .
$$

If $\chi_{1}$ denotes the trivial character, then $\alpha_{1}(A)=|A| /|G|=\delta(A)$. Altogether, we find

$$
\log Z_{A}(s)=\delta(A) \log \zeta_{L}(s)+\xi_{A}(s)+\eta_{A}(s),
$$

where

$$
\xi_{A}(s)=\sum_{i=2}^{h} \alpha_{i}(A) \log L\left(s, \chi_{i}\right)
$$

and $\eta_{A}$ denotes a function which is holomorphic and bounded in every halfplane $\operatorname{Re}(s) \geq \frac{1}{2}+\varepsilon, \varepsilon>0$. Note that $(s-1) \zeta_{L}(s)$ and $L\left(s, \chi_{i}\right), i>1$, are holomorphic and non-zero in $\mathcal{D}$. Thus, for $v \in \mathbb{C}$,

$$
\begin{aligned}
Z_{A}^{v}(s):= & \exp (-\delta(A) v \log (s-1) \\
& \left.+\delta(A) v \log \left((s-1) \zeta_{L}(s)\right)+v \xi_{A}(s)+v \eta_{A}(s)\right)
\end{aligned}
$$

gives the analytic continuation of $Z_{A}^{v}(s)$ to $\overline{\mathcal{D}}:=\mathcal{D} \backslash[\psi(0), 1]$. From (18) it follows that $\log Z_{A}(s) \ll \log _{2}(|t|+3)$ for $s \in \mathcal{D},|t| \geq \delta$. Hence, there is a constant $c(B)$ such that

$$
Z_{A}^{v}(s) \ll(\log |t|)^{c(B)}
$$

uniformly for $|v| \leq B, s \in \mathcal{D},|t| \geq \delta$. 
For $\operatorname{Re}(s)>1$ and $z=\left(z_{1}, \ldots, z_{d}\right) \in \mathbb{C}^{d}$ we write

$$
F(s, z)=G(s, z) \prod_{\nu=1}^{d} Z_{A_{\nu}}^{z_{\nu}}(s)
$$

where

$$
G(s, z)=\prod_{\nu=1}^{d} \prod_{\substack{\mathfrak{p} \nmid \mathfrak{d} \\(\mathfrak{p}, L / Q) \subseteq A_{\nu}}}\left(1+\frac{z_{\nu}}{N_{Q}(\mathfrak{p})^{s}-1}\right)\left(1-\frac{1}{N_{Q}(\mathfrak{p})^{s}}\right)^{z_{\nu}} .
$$

As a function of $s, G$ is analytic in $\operatorname{Re}(s)>1 / 2$, and uniformly bounded in $\operatorname{Re}(s) \geq 1 / 2+\varepsilon>1 / 2,\left|z_{\nu}\right| \leq B, 1 \leq \nu \leq d$ (cf. G. Tenenbaum [22, p. 201] for a more detailed argument). Thus (21) gives the analytic continuation of $F$ to $\overline{\mathcal{D}}$.

Let $x \geq 4, a=1+1 / \log x$ and set $T=\exp (\sqrt{\log x})-3$. Using Perron's formula and (20) we find, for an arbitrary $\varepsilon>0$,

$$
\begin{aligned}
\int_{0}^{x} S(u, z) d u & =\frac{1}{2 \pi i} \int_{a-i \infty}^{a+i \infty} F(s, z) x^{s+1} \frac{d s}{s(s+1)} \\
& =\frac{1}{2 \pi i} \int_{a-i T}^{a+i T} F(s, z) x^{s+1} \frac{d s}{s(s+1)}+O\left(x^{2} T^{-1+\varepsilon}\right) .
\end{aligned}
$$

Here and in the following, all estimates are uniform in $\left|z_{1}\right| \leq B, \ldots,\left|z_{d}\right| \leq B$. The constants implied in the $O$ - and $\ll$-notation may depend on $B$ and the field $L$.

Next, Cauchy's theorem is used to replace the path of integration by a path on the boundary of $\overline{\mathcal{D}}$. The new integration path is $\mathcal{G}_{1} \cup \mathcal{G}_{2} \cup \mathcal{H} \cup$ $\mathcal{G}_{3} \cup \mathcal{G}_{4}$, where $\mathcal{G}_{1}$ is the line connecting $a-i T$ with $\psi(T)-i T, \mathcal{G}_{2}$ is the curve $\psi(t)-i t, t$ running from $-T$ to $0, \mathcal{G}_{3}=-\overline{\mathcal{G}}_{2}, \mathcal{G}_{4}=-\overline{\mathcal{G}}_{1}$ and $\mathcal{H}$ is the Hankel type integration path connecting $\psi(0)$ with $1-\varrho$ (on this part of the path $\arg (s)=-\pi)$, turning around $s=1$ at a circle with radius $\varrho:=(2 \log x)^{-1}$ and connecting $1-\varrho$ with $\psi(0)$ (here $\arg (s)=\pi$ ). Using (20) we find that the contribution of the integral over $\mathcal{G}_{1}$ and $\mathcal{G}_{4}$ is $O\left(x^{2} T^{-2+\varepsilon}\right)$. Similarly, the contribution of the integrals over $\mathcal{G}_{2}$ and $\mathcal{G}_{3}$ is $O\left(x^{1+\sigma(T)}\right)=O\left(x^{2} \exp (-c \sqrt{\log x})\right)$. Hence, by (15),

$$
\int_{0}^{x} S(u, z) d u=\Phi(x, z)+O\left(x^{2} \exp (-c \sqrt{\log x})\right),
$$

where $0<c<1$ and

$$
\Phi(x, z)=\frac{1}{2 \pi i} \int_{\mathcal{H}} F(s, z) x^{s+1} \frac{d s}{s(s+1)} .
$$


Taylor series expansion in (19) yields

$$
Z_{A}^{v}(s)=(s-1)^{-\delta(A) v} e^{\beta(A) v}(1+O(|s-1|)) \quad(s \in \mathcal{H}, v \in \mathbb{C},|v| \leq B)
$$

with $\beta(A)=\delta(A) \operatorname{res}_{s=1} \zeta_{L}(s)+\xi_{A}(1)+\eta_{A}(1)$. It follows that, for $s \in \mathcal{H}$,

$$
\frac{1}{s} F(s, z)=H(z) \Gamma(w+1)(s-1)^{-w}+O\left(|s-1|^{1-\operatorname{Re}(w)}\right),
$$

where $w=\sum_{\nu=1}^{d} \delta_{\nu} z_{\nu}$ and

$$
H(z):=\frac{1}{\Gamma(w+1)} G(1, z) \prod_{\nu=1}^{d} e^{\beta\left(A_{\nu}\right) z_{\nu}} .
$$

Note that $H((0, \ldots, 0))=1$. Using $(23)$ we find

$$
\begin{aligned}
\frac{\partial^{2} \Phi}{\partial x^{2}}(x, z) & =\frac{1}{2 \pi i} \int_{\mathcal{H}} F(s, z) x^{s-1} d s \\
& \ll \int_{\mathcal{H}}|s-1|^{-\operatorname{Re}(w)}\left|x^{s-1}\right||d s| \ll(\log x)^{\operatorname{Re}(w)}
\end{aligned}
$$

and

$$
\begin{aligned}
\frac{\partial \Phi}{\partial x}(x, z) & =\frac{1}{2 \pi i} \int_{\mathcal{H}} F(s, z) x^{s} \frac{d s}{s} \\
& =H(z) w \Gamma(w) \frac{1}{2 \pi i} \int_{\mathcal{H}}(s-1)^{-w} x^{s} d s+O(R(x))
\end{aligned}
$$

Here

$$
\begin{aligned}
R(x) & =\int_{\mathcal{H}}|s-1|^{1-\operatorname{Re}(w)}\left|x^{s}\right||d s| \ll \int_{\psi(0)}^{1-\varrho}(1-\sigma)^{1-\operatorname{Re}(w)} x^{\sigma} d \sigma+x^{1+\varrho} \varrho^{2-\operatorname{Re}(w)} \\
& \ll x(\log x)^{\operatorname{Re}(w)-2}\left(\int_{1 / 2}^{\infty} u^{1-\operatorname{Re}(w)} e^{-u} d u+1\right) \ll x(\log x)^{\operatorname{Re}(w)-2} .
\end{aligned}
$$

By G. Tenenbaum [22, II.5, Corollary 2.1],

$$
\frac{1}{2 \pi i} \int_{\mathcal{H}}(s-1)^{-w} x^{s} d s=x(\log x)^{w-1}\left(\Gamma(w)^{-1}+O\left(x^{-\psi(0) / 2}\right)\right)
$$

uniformly for $|w| \leq B^{\prime}, B^{\prime}>0$. Hence

$$
\frac{\partial \Phi}{\partial x}(x, z)=w H(z) x(\log x)^{w-1}+O\left(x(\log x)^{\operatorname{Re}(w)-2}\right) .
$$


Using (22) and (25) we infer, with $h:=\exp (-(c / 2) \sqrt{\log x})$,

$$
\begin{aligned}
h^{-1} \int_{x}^{x+h} S(u, z) d u & =h^{-1}(\Phi(x+h, z)-\Phi(x, z))+O\left(x^{2} h\right) \\
& =\frac{\partial \Phi}{\partial x}(x, z)+h \int_{0}^{1}(1-t) \frac{\partial^{2} \Phi}{\partial x^{2}}(x+t h, z) d t+O\left(x^{2} h\right) \\
& =\frac{\partial \Phi}{\partial x}(x, z)+O\left(x^{2} h\right) .
\end{aligned}
$$

Together with (26) this implies

$$
h^{-1} \int_{x}^{x+h} S(u, z) d u=w H(z) x(\log x)^{w-1}+O\left(x(\log x)^{\operatorname{Re}(w)-2}\right) .
$$

Furthermore,

$$
S(x, z)=h^{-1} \int_{x}^{x+h} S(u, z) d u+O(D(x)),
$$

where, with $\mathbf{B}:=(B, \ldots, B) \in \mathbb{R}_{+}^{s}$,

$$
\begin{aligned}
D(x) & =h^{-1} \int_{x}^{x+h}|S(u, z)-S(x, z)| d u \\
& \leq h^{-1} \int_{x}^{x+h}(S(u, \mathbf{B})-S(x, \mathbf{B})) d u \\
& \leq h^{-1} \int_{x}^{x+h} S(u, \mathbf{B}) d u-h^{-1} \int_{x-h}^{x} S(u, \mathbf{B}) d u .
\end{aligned}
$$

Here we used the fact that $S(u, \mathbf{B}) \geq 0$ is non-decreasing in $u$. Applying (27) in (28) and (29) three times, we obtain

$$
S(x, z)=w H(z) x(\log x)^{w-1}+O\left(x(\log x)^{\operatorname{Re}(w)-2}\right)
$$

uniformly for $\left|z_{1}\right| \leq B, \ldots,\left|z_{d}\right| \leq B$. Remember that $w=\sum_{\nu=1}^{d} \delta_{\nu} z_{\nu}$.

Now a $d$-fold application of Cauchy's theorem yields

$$
\begin{aligned}
\pi(x) & =\pi_{K / Q}\left(x, r_{1}, \ldots, r_{d}\right) \\
& =\frac{1}{(2 \pi i)^{d}} \int_{C\left(\varrho_{1}\right)} \ldots \int_{C\left(\varrho_{d}\right)} S(x, z)\left(\prod_{\nu=1}^{d} z_{\nu}^{-r_{\nu}-1}\right) d z_{1} \ldots d z_{d} .
\end{aligned}
$$

Here $C\left(\varrho_{\nu}\right)$ denotes the positively oriented circle with radius $\varrho_{\nu}:=$ $\max \left(1, r_{\nu}\right) / X_{\nu}, X_{\nu}:=\delta_{\nu} \log _{2} x$, and center in the origin. Using (30) we find 


$$
\begin{aligned}
\pi(x)= & \frac{x}{\log x} \frac{1}{(2 \pi i)^{d}} \\
& \times \int_{C\left(\varrho_{1}\right)} \ldots \int_{C\left(\varrho_{d}\right)} \sum_{\mu=1}^{d} \delta_{\mu} z_{\mu} H(z) \prod_{\nu=1}^{d} e^{X_{\nu} z_{\nu}} z_{\nu}^{-r_{\nu}-1} d z_{\nu}+O\left(R_{1}(x)\right)
\end{aligned}
$$

with

$$
R_{1}(x) \ll \frac{x}{(\log x)^{2}} \prod_{\nu=1}^{d}\left(\varrho_{\nu}^{-r_{\nu}} \int_{0}^{2 \pi} e^{\max \left(1, r_{\nu}\right) \cos \varphi} d \varphi\right)
$$

The first of the two elementary bounds (cf. Tenenbaum [22, p. 204])

$$
\begin{aligned}
\int_{0}^{2 \pi} e^{x \cos \varphi} d \varphi \ll x^{-1 / 2} e^{x}, \\
\int_{0}^{2 \pi} e^{x \cos \varphi}(1-\cos \varphi) d \varphi \ll x^{-3 / 2} e^{x}
\end{aligned}
$$

and $n ! \sim \sqrt{2 \pi} n^{n+1 / 2} e^{-n}$ yield (recall $r=\sum_{\nu=1}^{d} r_{\nu}$ )

$$
\begin{aligned}
R_{1}(x) & \ll \frac{x}{(\log x)^{2}}\left(\log _{2} x\right)^{r} \prod_{\nu=1}^{d}\left(\delta_{\nu}^{r_{\nu}} \max \left(1, r_{\nu}\right)^{-r_{\nu}-1 / 2} e^{r_{\nu}}\right) \\
& \ll \frac{x}{(\log x)^{2}}\left(\log _{2} x\right)^{r} \prod_{\nu=1}^{d} \frac{\delta_{\nu}^{r_{\nu}}}{r_{\nu} !}
\end{aligned}
$$

To analyze the main term we remark that Taylor series expansion of $H$ at $z=0$ is sufficient to prove an asymptotic expansion of $\pi(x)$ with an error term of order $O\left(r\left(\log _{2} x\right)^{-1}\right)$. This is too weak to deduce a lower bound for $\pi(x)$ if $r \gg \log _{2} x$. Alternatively, we expand $H$ at $a=\left(a_{1}, \ldots, a_{d}\right)$ and choose $a$ in such a way that the contribution of the linear terms vanishes. Applying $F(1)=F(0)+F^{\prime}(0)+\int_{0}^{1}(1-t) F^{\prime \prime}(t) d t$ to $F(t)=H(a+t(z-a))$ we find

$$
H(z)=H(a)+\sum_{\kappa=1}^{d} \frac{\partial H}{\partial z_{\kappa}}(a)\left(z_{\kappa}-a_{\kappa}\right)+\sum_{\kappa, \iota=1}^{d}\left(z_{\kappa}-a_{\kappa}\right)\left(z_{\iota}-a_{\iota}\right) H_{\kappa, \iota}(z)
$$

with

$$
H_{\kappa, \iota}(z)=\int_{0}^{1}(1-t) \frac{\partial^{2} H}{\partial z_{\kappa} \partial z_{\iota}}(a+t(z-a)) d t \ll 1 .
$$

The contribution of the linear terms to $\pi(x)$ vanishes if we choose $a_{\nu}:=$ 
$(1-1 / r) r_{\nu} / X_{\nu}$. Indeed

$$
\begin{aligned}
& \frac{1}{(2 \pi i)^{d}} \int_{C\left(\varrho_{1}\right)} \ldots \int_{C\left(\varrho_{d}\right)} \sum_{\mu=1}^{d} \delta_{\mu} z_{\mu}\left(z_{\kappa}-a_{\kappa}\right) \prod_{\nu=1}^{d} e^{X_{\nu} z_{\nu}} z_{\nu}^{-r_{\nu}-1} d z_{\nu} \\
& =\left(\prod_{\nu=1}^{d} \frac{X_{\nu}^{r_{\nu}}}{r_{\nu} !}\right)\left(\sum_{\mu \neq \kappa} \delta_{\mu} \frac{r_{\mu}}{X_{\mu}}\left(\frac{r_{\kappa}}{X_{\kappa}}-a_{\kappa}\right)+\delta_{\kappa} \frac{r_{\kappa}}{X_{\kappa}}\left(\frac{r_{\kappa}-1}{X_{\kappa}}-a_{\kappa}\right)\right) \\
& =\left(\log _{2} x\right)^{r-1}\left(\prod_{\nu=1}^{d} \frac{\delta_{\nu}^{r_{\nu}}}{r_{\nu} !}\right)\left((r-1) \frac{r_{\kappa}}{X_{\kappa}}-r a_{\kappa}\right)=0 .
\end{aligned}
$$

The contribution of the second derivatives of $H$ to $\pi(x)$ is

$$
R_{2}(x):=\frac{x}{\log x} \sum_{\mu, \kappa, \iota=1}^{d} I_{\mu, \kappa, \iota}
$$

where

$$
\begin{aligned}
I_{\mu, \kappa, \iota}= & \frac{1}{(2 \pi i)^{d}} \\
& \times \int_{C\left(\varrho_{1}\right)} \ldots \int_{C\left(\varrho_{d}\right)} \delta_{\mu} z_{\mu}\left(z_{\kappa}-a_{\kappa}\right)\left(z_{\iota}-a_{\iota}\right) H_{\kappa, \iota}(z) \prod_{\nu=1}^{d} e^{X_{\nu} z_{\nu}} z_{\nu}^{-r_{\nu}-1} d z_{\nu} .
\end{aligned}
$$

If $a_{\nu}>0$ the integration path $C\left(\varrho_{\nu}\right)$ can be replaced by $C\left(a_{\nu}\right)$. Set $b_{\nu}=a_{\nu}$ if $a_{\nu}>0$ and $b_{\nu}=\varrho_{\nu}$ else. Since $H_{\kappa, \iota} \ll 1$ it follows that

$$
\begin{aligned}
I_{\mu, \kappa, \iota} \ll & \left(\prod_{\nu=1}^{d} b_{\nu}^{-r_{\nu}}\right) b_{\mu} b_{\kappa} b_{\iota} \\
& \times \int_{0}^{2 \pi} \ldots \int_{0}^{2 \pi}\left|1-I_{\left\{a_{\kappa}>0\right\}} e^{i \varphi_{\kappa}}\right|\left|1-I_{\left\{a_{\iota}>0\right\}} e^{i \varphi_{\iota}}\right| \prod_{\nu=1}^{d} e^{X_{\nu} b_{\nu} \cos \varphi_{\nu}} d \varphi_{\nu} .
\end{aligned}
$$

Using Cauchy's inequality and the bounds (31) we find

$$
I_{\mu, \kappa, \iota} \ll\left(\prod_{\nu=1}^{d} b_{\nu}^{-r_{\nu}}\right) b_{\mu} b_{\kappa} b_{\iota}\left(X_{\kappa} b_{\kappa}\right)^{-1 / 2}\left(X_{\iota} b_{\iota}\right)^{-1 / 2} \prod_{\nu=1}^{d}\left(X_{\nu} b_{\nu}\right)^{-1 / 2} e^{X_{\nu} b_{\nu}}
$$

Note that $X_{\kappa} b_{\kappa}=1$ if $a_{\kappa}=0$. It follows that

$$
R_{2}(x) \ll \frac{x}{\log x}\left(\prod_{\nu=1}^{d} b_{\nu}^{-r_{\nu}-1 / 2} X_{\nu}^{-1 / 2} e^{X_{\nu} b_{\nu}}\right) \sum_{\mu=1}^{d} b_{\mu}\left(\sum_{\kappa=1}^{d} b_{\kappa}^{1 / 2} X_{\kappa}^{-1 / 2}\right)^{2}
$$




$$
\begin{aligned}
& \ll \frac{x}{\log x}\left(\log _{2} x\right)^{r-3}\left(\prod_{\nu=1}^{d} \delta_{\nu}^{r_{\nu}}\left(X_{\nu} b_{\nu}\right)^{-r_{\nu}-1 / 2} e^{X_{\nu} b_{\nu}}\right) \\
& \quad \times \sum_{\mu=1}^{d} \max \left(1, r_{\mu}\right)\left(\sum_{\kappa=1}^{d} \max \left(1, r_{\kappa}\right)^{1 / 2}\right)^{2} \\
& \ll \frac{x}{\log x}\left(\log _{2} x\right)^{r-3}\left(\prod_{\nu=1}^{d} \frac{\delta_{\nu}^{r_{\nu}}}{r_{\nu} !}\right) r^{2} .
\end{aligned}
$$

Finally, the contribution of the term $H(a)$ to $\pi(x)$ is

$$
\frac{r x}{\log x}\left(\log _{2} x\right)^{r-1}\left(\prod_{\nu=1}^{d} \frac{\delta_{\nu}^{r_{\nu}}}{r_{\nu} !}\right) \mu\left(x, r_{1}, \ldots, r_{d}\right)
$$

where

$$
\mu\left(x, r_{1}, \ldots, r_{d}\right):=H\left(\frac{(r-1) r_{1}}{r \delta_{1} \log _{2} x}, \ldots, \frac{(r-1) r_{d}}{r \delta_{d} \log _{2} x}\right) .
$$

Altogether, we have proved

$$
\pi(x)=\left(\prod_{\nu=1}^{d} \frac{\delta_{\nu}^{r_{\nu}}}{r_{\nu} !}\right) \frac{r x}{\log x}\left(\log _{2} x\right)^{r-1}\left(\mu\left(x, r_{1}, \ldots, r_{d}\right)+O\left(\frac{r}{\left(\log _{2} x\right)^{2}}\right)\right) .
$$

Since $H(z)=1+O\left(\left|z_{1}\right|\right)+\cdots+O\left(\left|z_{d}\right|\right)$ and $H(z)>0$ for real $z_{1} \geq 0, \ldots, z_{d}$ $\geq 0$, we find $\mu\left(x, r_{1}, \ldots, r_{d}\right)=1+O\left(r\left(\log _{2} x\right)^{-1}\right)$ and $\mu\left(x, r_{1}, \ldots, r_{d}\right) \asymp 1$. This completes the proof of Theorem 2.

\section{Some special examples}

4.1. For $K=\mathbb{Q}$ and $m \geq 2$ arbitrary, Theorem 1 contains Soundararajan's result on the Piltz divisor problem established already in [19]:

$$
\begin{aligned}
& \Delta_{\mathbb{Q}, m}(x) \\
& \quad=\Omega\left((x \log x)^{1 / 2-1 / 2 m}\left(\log _{2} x\right)^{(1 / 2+1 / 2 m)\left(m^{2 m /(m+1)}-1\right)}\left(\log _{3} x\right)^{-3 / 4+1 / 4 m}\right),
\end{aligned}
$$

where $\Omega$ can be replaced by $\Omega_{+}$if $m \equiv 3(\bmod 8)$, and by $\Omega_{-}$if $m \equiv 7$ $(\bmod 8)$.

4.2. If $K$ is a normal extension of $\mathbb{Q}$ of degree $k \geq 2$, then $\delta_{\nu}=0$ for $1 \leq \nu<k$ and $\delta_{k}=\frac{1}{k}$ (cf. W. Narkiewicz [15, p. 357, Cor. 4]). For arbitrary $m \geq 1$, Theorem 1 applies with

$$
\kappa=\frac{1}{2} m(k m+1)(k m)^{-2 /(k m+1)}-\frac{1}{2}-\frac{1}{2 m} .
$$

The last exponent should be compared with Hafner's (see [4])

$$
\kappa^{\prime}=\frac{k m-1}{2 k} \log (k m)+\frac{1}{2}(m-1)\left(1+\frac{1}{k m}\right) .
$$


Note that, for $k m$ large, $\kappa \sim \frac{1}{2} k m^{2}$, while $\kappa^{\prime} \sim \frac{1}{2} m \log (k m)$. This case clearly contains that of a quadratic field $K$ : the result then reads

$$
\begin{aligned}
\Delta_{K, m}(x)=\Omega\left((x \log x)^{1 / 2-1 / 4 m}\left(\log _{2} x\right)^{\frac{1}{2} m(2 m+1)(2 m)^{-2 /(2 m+1)}-1 / 2-1 / 4 m}\right. & \left.\times\left(\log _{3} x\right)^{-3 / 4+1 / 8 m}\right) .
\end{aligned}
$$

Since $P(x)=4 \Delta_{\mathbb{Q}(i), 1}(x)$, this in turn contains the second line of $(2)$.

4.3. As the simplest example of a field $K$ which is not a normal extension of the rationals, let us consider a cubic field whose discriminant $D$ is not a perfect square (if the discriminant is a full square, the field $K$ is normal). In this case $L=K(\sqrt{D})$ is the minimal normal extension. Its Galois group is isomorphic to $S_{3}$, the symmetric group of three elements, and $H=\operatorname{Gal}(L / K)$ is a cyclic subgroup of order 2 . Using (4) we find $\delta_{1}=\frac{1}{2}$, $\delta_{2}=0$ and $\delta_{3}=\frac{1}{6}$. It follows that, for any $m \geq 1$,

$$
\Delta_{K, m}(x)=\Omega\left((x \log x)^{1 / 2-1 / 6 m}\left(\log _{2} x\right)^{\kappa}\left(\log _{3} x\right)^{-1}\right),
$$

with

$$
\kappa=\frac{1}{12} m^{(3 m-1) /(3 m+1)}(3 m+1)\left(3^{(3 m-1) /(3 m+1)}+1\right)-\frac{1}{2}-\frac{1}{6 m} .
$$

For $m=1$, this gives $\kappa=\frac{1}{3}(\sqrt{3}-1) \approx 0.2440$, while Hafner had only $\kappa^{\prime}=\frac{1}{6} \log 3 \approx 0.1831$. Similarly, for $m=2, \kappa=\frac{7}{12}\left(2^{5 / 7}+6^{5 / 7}-1\right) \approx 2.4714$, whereas $\kappa^{\prime}=\frac{5}{12} \log 12+\frac{7}{12} \approx 1.6187$.

4.4. As a last, more intrinsic example, let us consider $K=\mathbb{Q}(\alpha)$, where $\alpha \in \mathbb{C}$ is a zero of an (irreducible) polynomial $f$ over $\mathbb{Q}$ of degree seven with Galois group PSL $(3,2)$ (which is a simple group of order 168). In particular, $k=[K: \mathbb{Q}]=7$. Infinitely many number fields $K$ of this kind are known (see B. H. Matzat [14]), two of them being given by $f=X^{7}-7 X+3$ and $f=X^{7}-7 X^{3}+14 X^{2}-7 X+1$. There are exactly three non-empty sets $P_{\nu}$ belonging to $K$, namely $P_{1}, P_{3}$, and $P_{7}$, with corresponding Dirichlet densities $\delta_{1}=7 / 12, \delta_{3}=1 / 8$, and $\delta_{7}=1 / 168$. The set $P_{7}$ consists of those primes which split completely in $K$. The members of $P_{3}$ split into prime ideals whose degrees are given by the quintuple $(1,1,1,2,2)$. Finally, $P_{1}$ consists of two different types of primes whose decomposition is described by $(1,2,4)$ and $(1,3,3)$. It follows that, for any $m \geq 1$,

$$
\Delta_{K, m}(x)=\Omega\left((x \log x)^{1 / 2-1 / 14 m}\left(\log _{2} x\right)^{\kappa}\left(\log _{3} x\right)^{-5 / 4-1 / 28 m}\right),
$$

with

$$
\kappa=m^{(7 m-1) /(7 m+1)}(7 m+1)\left(\frac{7^{-2 /(7 m+1)}}{48}+\frac{3^{14 m /(7 m+1)}}{112}+\frac{1}{24}\right)-\frac{1}{2}-\frac{1}{14 m} .
$$

For $m=1$, this gives $\kappa=\frac{1}{42}\left(3^{11 / 4}+7^{3 / 4}-10\right) \approx 0.3528$, while Hafner had 
only

$$
\kappa^{\prime}=\frac{1}{56}(\log 7+9 \log 3) \approx 0.2113 .
$$

Similarly, for $m=2$,

$$
\kappa=\frac{1}{112}\left(45 \cdot 6^{13 / 15}+70 \cdot 2^{13 / 15}+5 \cdot 14^{13 / 15}-60\right) \approx 2.94196,
$$

whereas Hafner's $\kappa^{\prime}$ equals

$$
\frac{1}{336}(13 \log 7+117 \log 3+312 \log 2+180) \approx 1.63719 .
$$

\section{References}

[1] F. Fricker, Einführung in die Gitterpunktlehre, Birkhäuser, Basel, 1982.

[2] J. L. Hafner, New Omega theorems for two classical lattice point problems, Invent. Math. 63 (1981), 181-186.

[3] -, On the average order of a class of arithmetical functions, J. Number Theory 15 (1982), 36-76.

[4] - The distribution and average order of the coefficients of Dedekind $\zeta$ functions, ibid. 17 (1983), 183-190.

[5] G. H. Hardy, On Dirichlet's divisor problem, Proc. London Math. Soc. (2) 15 (1916), $1-25$.

[6] M. N. Huxley, Exponential sums and lattice points II, Proc. London Math. Soc. (3) 66 (1993), 279-301.

[7] -, Area, Lattice Points, and Exponential Sums, London Math. Soc. Monogr. (N.S.) 13, Oxford Univ. Press, Oxford, 1996.

[8] —, Exponential sums and lattice points III, Proc. London Math. Soc. (3) 87 (2003), 591-609.

[9] A. Ivić, The Riemann Zeta-Function, Wiley, New York, 1985.

[10] G. J. Janusz, Algebraic Number Fields, Academic Press, New York, 1973.

[11] E. Krätzel, Lattice Points, Deutscher Verlag Wiss., Berlin, 1988.

[12] —, Analytische Funktionen in der Zahlentheorie, Teubner, Stuttgart, 2000.

[13] J. C. Lagarias and A. M. Odlyzko, Effective versions of the Chebotarev density theorem, in: A. Fröhlich (ed.), Algebraic Number Fields, Academic Press, London, 1977, 409-464.

[14] B. H. Matzat, Konstruktion von Zahl- und Funktionenkörpern mit vorgegebener Galoisgruppe, J. Reine Angew. Math. 349 (1984), 179-220.

[15] W. Narkiewicz, Elementary and Analytic Theory of Algebraic Numbers, 2nd ed., Springer, Berlin, and PWN, Warszawa, 1990.

[16] W. G. Nowak, On the distribution of integer ideals in algebraic number fields, Math. Nachr. 161 (1993), 59-74.

[17] —, On the general asymmetric divisor problem, Abh. Math. Sem. Univ. Hamburg 65 (1995), 259-276.

[18] E. de Shalit, Artin L functions, in: P. Bernstein and S. Gelbart (eds.), An Introduction to the Langlands Program, Birkhäuser, Boston, 2003, 73-87.

[19] K. Soundararajan, Omega results for the divisor and circle problems, Internat. Math. Res. Notices 2003, no. 36, 1987-1998. 
[20] G. Szegő und A. Walfisz, Über das Piltzsche Teilerproblem in algebraischen Zahlkörpern, erste Abhandlung, Math. Z. 26 (1927), 138-156.

[21] - - - Über das Piltzsche Teilerproblem in algebraischen Zahlkörpern, zweite Abhandlung, ibid., 467-486.

[22] G. Tenenbaum, Introduction to Analytic and Probabilistic Number Theory, Cambridge Stud. Adv. Math. 46, Cambridge Univ. Press, Cambridge, 1995.

Kurt Girstmair

Institut für Mathematik

Universität Innsbruck

Technikerstraße 25

6020 Innsbruck, Austria

E-mail: Kurt.Girstmair@uibk.ac.at

Web: http://mathematik.uibk.ac.at/users/girstmai/

Manfred Kühleitner \& Werner Georg Nowak

Institut für Mathematik

Department für Integrative Biologie

Universität für Bodenkultur Wien

Gregor Mendel-Straße 33

1180 Wien, Austria

E-mail: kleitner@edv1.boku.ac.at

nowak@mail.boku.ac.at

Web: http://www.boku.ac.at/math/nth.html
Wolfgang Müller Institut für Statistik Technische Universität Graz 8010 Graz, Austria E-mail: mueller@stat.tu-graz.ac.at

Received on 31.8.2004

and in revised form on 25.10.2004 